\title{
Intraocular Pressure Reduction in a Pigmentary Glaucoma Model by Goniotome
}

\section{Ab Interno Trabeculectomy}

Chao Wang, ${ }^{1,2,3}$ Yalong Dang, ${ }^{1}$ Priyal Shah, ${ }^{1}$ Hamed Esfandiari, ${ }^{1}$ Ying Hong,${ }^{1,4}$ Ralitsa Loewen, ${ }^{1}$ Susannah

Waxman, ${ }^{1}$ Sarah Atta, ${ }^{1}$ Xiaobo Xia, ${ }^{2 *}$ Nils A. Loewen ${ }^{1 *}$

1: Department of Ophthalmology, University of Pittsburgh Medical Center, Pittsburgh, Pennsylvania, United States

2: Department of Ophthalmology, Xiangya Hospital, Central South University, Changsha, Hunan, China.

3: The Third Xiangya Hospital of Central South University, Changsha, Hunan, China.

4: Department of Ophthalmology, Peking University Third Hospital, Beijing, China.

* corresponding authors 


\section{Abstract}

Purpose: To investigate whether microsurgical excision of trabecular meshwork (TM) in an ex vivo pigmentary glaucoma model can normalize the hypertensive phenotype.

Methods: Eight eyes of a porcine pigmentary glaucoma model underwent $90^{\circ}$ of microsurgical TM excision with an aspirating dual-blade (Goniotome (G)). 24 hours later, an additional $90^{\circ}$ of TM were removed. Anterior segments with sham surgeries served as the control (C). Outflow facility and intraocular pressure (IOP) were analyzed. Histology with hematoxylin and eosin (H\&E) was obtained.

Results: After the first $90^{\circ} \mathrm{TM}$ excision, IOP was significantly lower in $\mathrm{G}(10.23 \pm 2.39 \mathrm{mmHg}, \mathrm{n}=7)$ than $\mathrm{C}$ (20.04 $\pm 1.97 \mathrm{mmHg}, \mathrm{n}=8, P<0.01)$. Outflow facility in $\mathrm{G}(0.38 \pm 0.07 \mu \mathrm{l} / \mathrm{min} / \mathrm{mmHg})$ was higher than $\mathrm{C}(0.16 \pm 0.02$ $\mu \mathrm{l} / \mathrm{min} / \mathrm{mmHg}, \mathrm{P}<0.01)$. After the second $90^{\circ} \mathrm{TM}$ excision, IOP in $\mathrm{G}(6.46 \pm 0.81 \mathrm{mmHg}, \mathrm{n}=7)$ was significantly lower than $\mathrm{C}(20.25 \pm 1.66 \mathrm{mmHg}, \mathrm{n}=8, P<0.001)$, while the outflow facility in $\mathrm{G}(0.50 \pm 0.05 \mu \mathrm{l} / \mathrm{min} / \mathrm{mmHg}, \mathrm{n}=7)$ was higher than $C(0.16 \pm 0.01 \mu \mathrm{l} / \mathrm{min} / \mathrm{mmHg}, \mathrm{n}=8, P<0.001)$. Compared to the first excision, excision of an additional $90^{\circ}$ did not change of IOP $(P=0.20)$ or outflow facility $(P=0.17)$ further.

Conclusion: Excision of $90^{\circ}$ of TM in a pigmentary glaucoma model using an aspirating dual-blade decreased IOP and increased outflow facility.

Translational Relevance: Microsurgical TM excision over $90^{\circ}$ can effectively restore outflow in pigmentary glaucoma.

Keywords: Pigmentary glaucoma; trabecular meshwork; Goniotome; intraocular pressure; outflow facility. 


\section{Introduction}

Pigment dispersion syndrome (PDS) can lead to pigmentary glaucoma (PG), a form of secondary open-angle glaucoma, which often affects nearsighted individuals in their 30 s to $40 \mathrm{~s}^{1,2}$ The prevalence of PDS is as high as $2.5 \%$ in the general population and incurs a risk of $15 \%$ of $P G$ within 15 years. ${ }^{3}$ Mid-peripheral iris transillumination defects can be seen early in the course of the disease, which can progress to more extensive iris atrophy. The cellular debris released by this tissue contains pigment granules that accumulate in the trabecular meshwork (TM), on the corneal endothelium in the form of the Krukenberg spindle, on the lens surface, and elsewhere. ${ }^{4}$ Cytoskeletal and structural TM changes ${ }^{5}$ make PG less responsive to medications or laser than other open angle glaucomas. ${ }^{2}$ Recent evidence points towards a central role of RhoA in regulating the cytoskeleton, motility, and phagocytosis of TM cells., ${ }^{5,6}$ Ocular hypertension emerges as soon as cytoskeletal changes occur and before phagocytosis declines. ${ }^{7}$

Trabecular ablation, for instance, in Trabectome surgery (Neomedix Corp., Tustin, California, United States), can be effective in a range of glaucoma disease stages ${ }^{8-13}$ which makes it well suited for PG. Ab interno angle surgeries rely on maintaining the anterior chamber either passively with a viscoelastic device ${ }^{14,15}$ or actively, using an irrigation and aspiration (I\&A) system. ${ }^{16,17}$ The advantages of a clear angle view and anterior chamber stability have recently become more readily available with an I\&A-equipped dual-blade (Goniotome, Neomedix Corp., Tustin, California, United States) that does not require a high-frequency generator to molecularize the TM and instead excises a strip. ${ }^{14,18}$ These features and its ability to excise TM in a controlled environment and to harvest it non-destructively make it also useful for glaucoma research.

We recently found that a Rho-kinase inhibitor can relax the contracted cytoskeleton and normalize outflow in an ex vivo PG model. ${ }^{19}$ In the present study, we hypothesized that trabecular excision with an I\&A-equipped dual-blade device could also restore outflow by removing the pathology. We have extensive experience studying outflow in experimental systems, ${ }^{20,21}$ including gene transfer, ${ }^{22-28}$ disease modeling ${ }^{5,7,19}$ and surgical outflow enhancement, ${ }^{14,15,18,29-32}$ but this is the first study of a microsurgical intervention for glaucoma in an ex vivo model of glaucoma. 


\section{Methods}

\section{Pig eye perfusion culture and pigmentary dispersion glaucoma model}

Sixteen porcine eyes were obtained from a local abattoir (Thoma Meat Market, Saxonburg, PA) as left-right matched pairs and were processed within two hours of sacrifice. Approval by the Institutional Animal Care and Use Committee was not required because no animals were sacrificed for the purpose of this study. After removal of extraocular tissues, the eyes were decontaminated in 5\% povidone-iodine solution (3955-16, Ricca Chemical Company, Arlington, TX 786012) for two minutes and irrigated three times with phosphate buffered saline (PBS). The posterior segment, lens, iris and ciliary body were carefully removed. Anterior segments with intact TM were mounted in perfusion dishes as described before. ${ }^{33}$ The perfusion media consisted of Dulbecco's modified Eagle media (DMEM, SH30284, HyClone, GE Healthcare, UK) supplemented with $1 \%$ fetal bovine serum (FBS, 10438026, Thermo Fisher Scientific, Waltham, MA) and 1\% antibiotic/antimycotic (15240062, Thermo Fisher Scientific, Waltham, MA) at a constant rate of $3 \mu \mathrm{l} / \mathrm{min}$ using a microinfusion pump (PHD 22/2000; Harvard Apparatus, Holliston, MA).

A suspension of pigment granules was generated with freeze-thaw cycles as described previously. ${ }^{5}$ In brief, the irises of 10 decontaminated porcine eyes were isolated, frozen at $-80 \mathrm{C}^{\circ}$ for two hours and thawed at room temperature for 2 hours. This process was performed twice to lyse cells and release pigment granules. The lysate was suspended in $15 \mathrm{~mL}$ PBS and aspirated and expelled 20 times to promote further pigment release. After filtering through a 70- $\mu$ m cell strainer (431751, Corning Incorporated, Durham, NC), the suspension was centrifuged at $3000 \mathrm{rpm}$ for 15 minutes. The supernatant was discarded, and the pigment was resuspended in $15 \mathrm{ml}$ PBS. Centrifugation and resuspension steps were repeated four times, and the pigment pellet was resuspended in $4 \mathrm{~mL}$ PBS for pigment stock solution. The stock solution was diluted 1000 fold and concentration was determined with a hemocytometer, using 600x magnification (Eclipse TE200-E, Nikon Instruments Inc., Melville, NY). Pigment granules were added to perfusion media after 48h of IOP stabilization, at a concentration of $1.67 \times 107$ particles $/ \mathrm{mL}$. The IOP was measured with pressure transducers (SP844; MEMSCAP, Skoppum, Norway) and recorded every two minutes (LabChart, ADInstruments, Colorado Springs, $\mathrm{CO})$. 


\section{Trabecular meshwork excision}

When the hypertensive plateau was established, the TM in experimental group G was excised with the Goniotome (Neomedix Corp., Tustin, CA, United States, Figure 1). Control eyes in group C did not undergo Goniotome surgery. All procedures were performed by a single surgeon (NAL) with comprehensive experience in ab interno trabeculectomy. The anterior segments were positioned under a surgical microscope (S4, Carl Zeiss Meditec, Jena, Germany), the tip of the instrument was inserted into the TM and aspiration was started. This caused the serrated dual-blades to engage and excise the TM. The excision was continued $90^{\circ}$ to the left, and the excised TM strip was cut by angulating the tip of the Goniotome. After 24 hours, an additional $90^{\circ}$ of the TM was removed using the same procedure with an excision path in the opposite direction.

\section{Histology}

After the perfusion culture experiments, the anterior segments were fixed with $4 \%$ paraformaldehyde for 24 hours, rinsed, embedded in paraffin, and sectioned at a thickness of 6 microns. Hematoxylin and eosin staining was performed for gross histological evaluation.

\section{Statistics}

Data were reported as the mean \pm standard error unless stated otherwise. A one-way ANOVA was used to compare the data for different groups at individual time points. Paired t-tests were used for an in-group comparison of IOP and outflow facility before and after treatment. (PASW 18.0, SPSS, Armonk, New York, United States). Differences were considered statistically significant for $P<0.05$. IOPs were averaged over 6 hours following excision. Media and dish handling were scheduled to occur outside of these periods to avoid artifacts. The outflow facility was computed using the Goldmann equation assuming that the episcleral venous pressure is near zero, $\mathrm{Po}=(\mathrm{F} / \mathrm{C})+\mathrm{Pv}(\mathrm{Po}$ : IOP, F: rate of aqueous formation, $\mathrm{C}$ : outflow facility, Pv: episcleral venous pressure). 


\section{Results}

Sixteen porcine eyes were used (eight eyes in G, and eight eyes in C). One eye in G was excluded due to a transducer error. After $48 \mathrm{~h}$ of perfusion, a stable baseline IOP was achieved $(10.65 \pm 1.29, \mathrm{n}=15)$, and the pigment was added to the perfusion system to establish the pigmentary glaucoma model. After $48 \mathrm{~h}$ of pigment perfusion, IOP was again stable but significantly increased $(15.92 \pm 1.63, n=15, P<0.001)$. The first TM excision in $\mathrm{G}$ reduced the IOP to $10.23 \pm 2.39 \mathrm{mmHg}(n=7) 24$ hours later compared to $20.04 \pm 1.97 \mathrm{mmHg}$ in $\mathrm{C}(\mathrm{n}=8$, $P<0.01)$. The second TM excision, now had an average in $G$ of only $6.46 \pm 0.81 \mathrm{mmHg},(n=7, P=0.20)$ compared to $20.25 \pm 1.66 \mathrm{mmHg}(\mathrm{n}=8, \mathrm{P}<0.001)$ in $\mathrm{C}$ (Figure 2).

After $48 \mathrm{~h}$ of perfusion, baseline outflow facility was stable $(0.41 \pm 0.09, \mathrm{n}=15)$. After $48 \mathrm{~h}$ of pigment perfusion, outflow facility of $P G(0.17 \pm 0.02, n=15, P<0.05)$ was significantly decreased from baseline. 24 hours after the first $90^{\circ}$ of $\mathrm{TM}$ excision, outflow facility in $\mathrm{G}(0.38 \pm 0.07 \mu \mathrm{l} / \mathrm{min} / \mathrm{mmHg}, \mathrm{n}=7)$ was higher than $\mathrm{C}$ $(0.16 \pm 0.02 \mu \mathrm{l} / \mathrm{min} / \mathrm{mmHg}, \mathrm{n}=8, \mathrm{P}<0.01) .24$ hours after the second TM excision, outflow facility in $\mathrm{G}(0.50 \pm 0.05$ $\mu \mathrm{l} / \mathrm{min} / \mathrm{mmHg}, \mathrm{n}=7)$ was higher than $\mathrm{C}(0.16 \pm 0.01 \mu \mathrm{l} / \mathrm{min} / \mathrm{mmHg}, \mathrm{n}=8, \mathrm{P}<0.001)$, but there was no significant further increase in outflow facility after second TM excision (Figure 3), compared with the outflow facility after first TM excision $(P=0.17)$.

The control TM presented as thick, multilayered structure composed of the uveal meshwork (box with solid line, Figure 4A), the corneoscleral meshwork (box with dashed lines, Figure 4A) and the juxtacanalicular meshwork (solid line, Figure 4A). Pigs have an angular aqueous plexus without a single lumen Schlemm's canal characteristic for primate eyes. Instead, smaller, Schlemm's canal-like segments (SCLS) adjacent to the juxtacanalicular meshwork could be made out. The normal TM was lightly-pigmented (Figure 4A). As seen before, ${ }^{5,7}$ pigment granules were apparent in the TM at a density far below the amount expected to cause a physical obstruction after perfusion with pigment-supplemented media for 48 hours. The pigment granules were seen throughout the TM (Figure 4B, yellow arrows). After the TM excision with the Goniotome, a full thickness portion of TM appeared removed without damage to the adjacent sclera or corneal endothelium (Figure 4C). In comparison to the smaller human TM that can be removed in its entirety, remnants of adjacent TM remained. 


\section{Discussion}

This is the first study of a microincisional intervention in an ex vivo model of glaucoma. We found that trabecular excision with a novel dual-blade device, the Goniotome, induces a 52.5\% IOP reduction after only $90^{\circ}$ of TM are removed. Additional TM excision did not significantly lower the IOP. This finding agrees with the clinical observation in human eyes that the length of TM ablation beyond $90^{\circ}$ does not lower IOP more even though it might improve the chances of successfully connecting to working collector channels. ${ }^{34,35}$ Pig eyes do not have a single, contiguous Schlemm's canal yet a circumferential flow pattern can be observed here as well. ${ }^{21,29}$

The trabecular meshwork and the inner wall of Schlemm's canal are sites of outflow resistance in open angle glaucoma, but they account for only a fraction of conventional outflow resistance. ${ }^{36}$ The resistance downstream of the TM appears to be responsible for a pressure higher than the episcleral venous pressure of approximately $8 \mathrm{mmHg}$. It causes the IOP to be near $16 \mathrm{mmHg}$ in average after ab interno trabeculectomy, but the postoperative IOP is positively correlated to a higher baseline pressure indicating the presence of an additional flow resistance that is not yet identified. ${ }^{37}$

In contrast, secondary open angle glaucomas are caused by pathologies mostly limited to the TM and respond well to ab interno trabeculectomy. ${ }^{38-41}$ Pigmentary glaucoma is such a secondary glaucoma and tends to present with a relatively high mean IOP of $30 \mathrm{mmHg}$ with a range from 24 to $56 \mathrm{mmHg}{ }^{3,42}$ The mechanism of outflow compromise in PG is not a simple mechanical obstruction but appears to be due to a rearrangement and contraction of the TM actin cytoskeleton. ${ }^{7}$ RhoA signaling plays a central role in the regulation of the TM actin cytoskeleton, of tight junction formation, phagocytosis, and cell motility. ${ }^{5,7}$ The phagocytic overload eventually leads to TM cell death, and collapse and fusion of denuded trabecular beams. ${ }^{43,44}$ Non-surgical treatment of PG is challenging, ${ }^{2,45}$ and in the past, about $35 \%$ of all PG patients needed traditional filtering surgery to control IOP ${ }^{46}$ The safety and efficacy of ab interno trabeculectomy are especially attractive for younger patients. As seen in this model, the continued presence of pigment dispersion does not appear to block collector channel orifices with a subsequent IOP rise at least in the short term. 
There are several limitations to our study. This is an ex vivo model, so immunologic responses and wound healing processes that could affect the postoperative IOP are not taken into account. The observation time as relatively short. Moreover, there are anatomical differences between human and porcine eyes that may result in a different IOP response.

In conclusion, in this porcine ex vivo PG model, Goniotome surgery caused a $52.5 \%$ IOP reduction after removal of $90^{\circ}$ of $\mathrm{TM}$.

\section{Acknowledgements}

We acknowledge support from K08 K08-EY022737(NAL), from NIH CORE Grant P30 EY08098 to the Department of Ophthalmology, from the Initiative to Cure Glaucoma of the Eye and Ear Foundation of Pittsburgh(NAL), the Wiegand Fellowship (YD) and an unrestricted grant from Research to Prevent Blindness, New York, NY, an unrestricted grant from the Third Xiangya Hospital of Central South University for studying at the University of Pittsburgh (CW). 


\section{References}

1. De Moraes CG, Susanna R Jr. Glaucomas: Pigment Dispersion Syndrome, Pigmentary Glaucoma, and Angle Recession Glaucoma. In: Giaconi JA, Law SK, Nouri-Mahdavi K, Coleman AL, Caprioli J, eds. Pearls of Glaucoma Management. Springer Berlin Heidelberg; 2016:419-430.

2. Niyadurupola N, Broadway DC. Pigment dispersion syndrome and pigmentary glaucoma--a major review. Clin Experiment Ophthalmol. 2008;36(9):868-882.

3. Siddiqui Y, Ten Hulzen RD, Cameron JD, Hodge DO, Johnson DH. What is the risk of developing pigmentary glaucoma from pigment dispersion syndrome? Am J Ophthalmol. 2003;135(6):794-799.

4. Ritch R, Steinberger D, Liebmann JM. Prevalence of pigment dispersion syndrome in a population undergoing glaucoma screening. Am J Ophthalmol. 1993;115(6):707-710.

5. Dang Y, Waxman S, Wang C, Loewen RT, Sun M, Loewen NA. A porcine ex vivo model of pigmentary glaucoma. Sci Rep. 2018;8(1):5468.

6. Wang C, Dang Y, Loewen RT, et al. Impact of Pigment Dispersion on Trabecular Meshwork Cells. Preprints. 2018;2018(2018040007):1-27.

7. Dang Y, Waxman S, Wang C, Shah P, Loewen RT, Loewen NA. Intraocular pressure elevation precedes a phagocytosis decline in a model of pigmentary glaucoma. F1000Res. 2018;7.

doi:10.12688/f1000research.13797.1

8. Bussel II, Kaplowitz K, Schuman JS, Loewen NA, Trabectome Study Group. Outcomes of ab interno trabeculectomy with the trabectome after failed trabeculectomy. Br J Ophthalmol. 2015;99(2):258-262.

9. Parikh HA, Bussel II, Schuman JS, Brown EN, Loewen NA. Coarsened Exact Matching of Phaco-Trabectome to Trabectome in Phakic Patients: Lack of Additional Pressure Reduction from Phacoemulsification. PLoS One. 2016;11(2):e0149384.

10. Neiweem AE, Bussel II, Schuman JS, Brown EN, Loewen NA. Glaucoma Surgery Calculator: Limited Additive Effect of Phacoemulsification on Intraocular Pressure in Ab Interno Trabeculectomy. PLoS One. 2016;11(4):e0153585.

11. Roy P, Loewen RT, Dang Y, Parikh HA, Bussel II, Loewen NA. Stratification of phaco-trabectome surgery results using a glaucoma severity index in a retrospective analysis. BMC Ophthalmol. 2017;17(1):30.

12. Loewen RT, Roy P, Parikh HA, Dang Y, Schuman JS, Loewen NA. Impact of a Glaucoma Severity Index on Results of Trabectome Surgery: Larger Pressure Reduction in More Severe Glaucoma. PLoS One. 2016;11(3):e0151926.

13. Dang Y, Roy P, Bussel II, Loewen RT, Parikh H, Loewen NA. Combined analysis of trabectome and phaco-trabectome outcomes by glaucoma severity. F1000Res. 2016;5:762.

14. Wang C, Dang Y, Waxman S, Xia X, Weinreb RN, Loewen NA. Angle stability and outflow in dual blade ab interno trabeculectomy with active versus passive chamber management. PLoS One.

2017;12(5):e0177238.

15. Parikh HA, Loewen RT, Roy P, Schuman JS, Lathrop KL, Loewen NA. Differential Canalograms Detect Outflow Changes from Trabecular Micro-Bypass Stents and Ab Interno Trabeculectomy. Sci Rep. 
$2016 ; 6: 34705$.

16. Kaplowitz K, Schuman JS, Loewen NA. Techniques and outcomes of minimally invasive trabecular ablation and bypass surgery. Br J Ophthalmol. 2014;98(5):579-585.

17. Kaplowitz K, Bussel I, Loewen NA. Minimally Invasive and Nonpenetrating Glaucoma Surgeries. In: Yanoff M, Duker JS, eds. Ophthalmology: Expert Consult: Online and Print. Elsevier - Health Sciences Division; 2013:1133-1146.

18. Dang $\mathrm{Y}$, Wang $\mathrm{C}$, Shah $\mathrm{P}$, et al. Outflow Enhancement by Three Different ab Interno Trabeculectomy Procedures in a Porcine Anterior Segment Model. Preprints. February 2018.

doi:10.20944/preprints201802.0028.v1

19. Dang Y, Wang C, Shah P, Waxman S, Loewen RT, Loewen NA. Ocular Hypotension, Actin Stress Fiber Disruption and Phagocytosis Increase by RKI-1447, a Rho-Kinase Inhibitor. Preprints. February 2018. doi:10.20944/preprints201802.0026.v1

20. Fautsch MP, Bahler CK, Vrabel AM, et al. Perfusion of his-tagged eukaryotic myocilin increases outflow resistance in human anterior segments in the presence of aqueous humor. Invest Ophthalmol Vis Sci. 2006;47(1):213-221.

21. Loewen RT, Brown EN, Roy P, Schuman JS, Sigal IA, Loewen NA. Regionally Discrete Aqueous Humor Outflow Quantification Using Fluorescein Canalograms. PLoS One. 2016;11(3):e0151754.

22. Loewen RT, Roy P, Park DB, et al. A Porcine Anterior Segment Perfusion and Transduction Model With Direct Visualization of the Trabecular Meshwork. Invest Ophthalmol Vis Sci. 2016;57(3):1338-1344.

23. Dang Y, Loewen R, Parikh HA, Roy P, Loewen NA. Gene transfer to the outflow tract. Exp Eye Res. April 2016:044396.

24. Zhang Z, Dhaliwal AS, Tseng $\mathrm{H}$, et al. Outflow tract ablation using a conditionally cytotoxic feline immunodeficiency viral vector. Invest Ophthalmol Vis Sci. 2014;55(2):935-940.

25. Loewen N, Fautsch MP, Peretz M, et al. Genetic modification of human trabecular meshwork with lentiviral vectors. Hum Gene Ther. 2001;12(17):2109-2119.

26. Loewen N, Bahler C, Teo W-L, et al. Preservation of aqueous outflow facility after second-generation FIV vector-mediated expression of marker genes in anterior segments of human eyes. Invest Ophthalmol Vis Sci. 2002;43(12):3686-3690.

27. Khare PD, Loewen N, Teo W, et al. Durable, safe, multi-gene lentiviral vector expression in feline trabecular meshwork. Mol Ther. 2008;16(1):97-106.

28. Loewen N, Fautsch MP, Teo W-L, Bahler CK, Johnson DH, Poeschla EM. Long-term, targeted genetic modification of the aqueous humor outflow tract coupled with noninvasive imaging of gene expression in vivo. Invest Ophthalmol Vis Sci. 2004;45(9):3091-3098.

29. Loewen RT, Brown EN, Scott G, Parikh H, Schuman JS, Loewen NA. Quantification of Focal Outflow Enhancement Using Differential Canalograms. Invest Ophthalmol Vis Sci. 2016;57(6):2831-2838.

30. Fallano K, Bussel I, Kagemann L, Lathrop KL, Loewen N. Training strategies and outcomes of ab interno trabeculectomy with the trabectome. F1000Res. 2017;6:67.

31. Dang $\mathrm{Y}$, Waxman S, Wang $\mathrm{C}$, et al. Rapid learning curve assessment in an ex vivo training system for microincisional glaucoma surgery. Sci Rep. 2017;7(1):1605.

32. Oatts JT, Zhang Z, Tseng H, Shields MB, Sinard JH, Loewen NA. In vitro and in vivo comparison of two 
suprachoroidal shunts. Invest Ophthalmol Vis Sci. 2013;54(8):5416-5423.

33. Dang $\mathrm{Y}$, Waxman S, Wang C, et al. Freeze-thaw decellularization of the trabecular meshwork in an ex vivo eye perfusion model. PeerJ. 2017;5:e3629.

34. Khaja HA, Hodge DO, Sit AJ. Trabectome Ablation Arc Clinical Results and Relation to Intraocular Pressure. Invest Ophthalmol Vis Sci. 2008;49(13):4191-4191.

35. Hunter KS, Fjield T, Heitzmann H, Shandas R, Kahook MY. Characterization of micro-invasive trabecular bypass stents by ex vivo perfusion and computational flow modeling. Clin Ophthalmol. 2014;8:499-506.

36. Tamm ER, Ethier CR. Current aspects of aqueous humor dynamics and glaucoma. Exp Eye Res. 2009;88(4):618-619.

37. Parikh HA, Bussel II, Schuman JS, Brown EN, Loewen NA. Coarsened Exact Matching of Phaco-Trabectome to Trabectome in Phakic Patients: Lack of Additional Pressure Reduction from Phacoemulsification. PLoS One. 2016;11(2):e0149384.

38. Ting JLM, Damji KF, Stiles MC, Trabectome Study Group. Ab interno trabeculectomy: outcomes in exfoliation versus primary open-angle glaucoma. J Cataract Refract Surg. 2012;38(2):315-323.

39. Kaplowitz K, Loewen NA. Trabectome-Mediated Ab Interno Trabeculectomy for Secondary Glaucoma or as a Secondary Procedure. In: Advanced Glaucoma Surgery. Essentials in Ophthalmology. Springer, Cham; 2015:15-29.

40. Ngai P, Kim G, Chak G, Lin K, Maeda M, Mosaed S. Outcome of primary trabeculotomy ab interno (Trabectome) surgery in patients with steroid-induced glaucoma. Medicine . 2016;95(50):e5383.

41. Dang Y, Kaplowitz K, Parikh HA, et al. Steroid-induced glaucoma treated with trabecular ablation in a matched comparison with primary open-angle glaucoma. Clin Experiment Ophthalmol. 2016;44(9):783-788.

42. Sugar HS. Pigmentary glaucoma. A 25-year review. Am J Ophthalmol. 1966;62(3):499-507.

43. Alvarado JA, Murphy CG. Outflow obstruction in pigmentary and primary open angle glaucoma. Arch Ophthalmol. 1992;110(12):1769-1778.

44. Gottanka J, Johnson DH, Grehn F, Lütjen-Drecoll E. Histologic findings in pigment dispersion syndrome and pigmentary glaucoma. J Glaucoma. 2006;15(2):142-151.

45. Ritch R, Campbell DG, Camras C. Initial treatment of pigmentary glaucoma. J Glaucoma. 1993;2(1):44-49.

46. Lehto I. Long-term follow up of argon laser trabeculoplasty in pigmentary glaucoma. Ophthalmic Surg. 1992;23(9):614-617. 


\section{Figures}

Figure 1

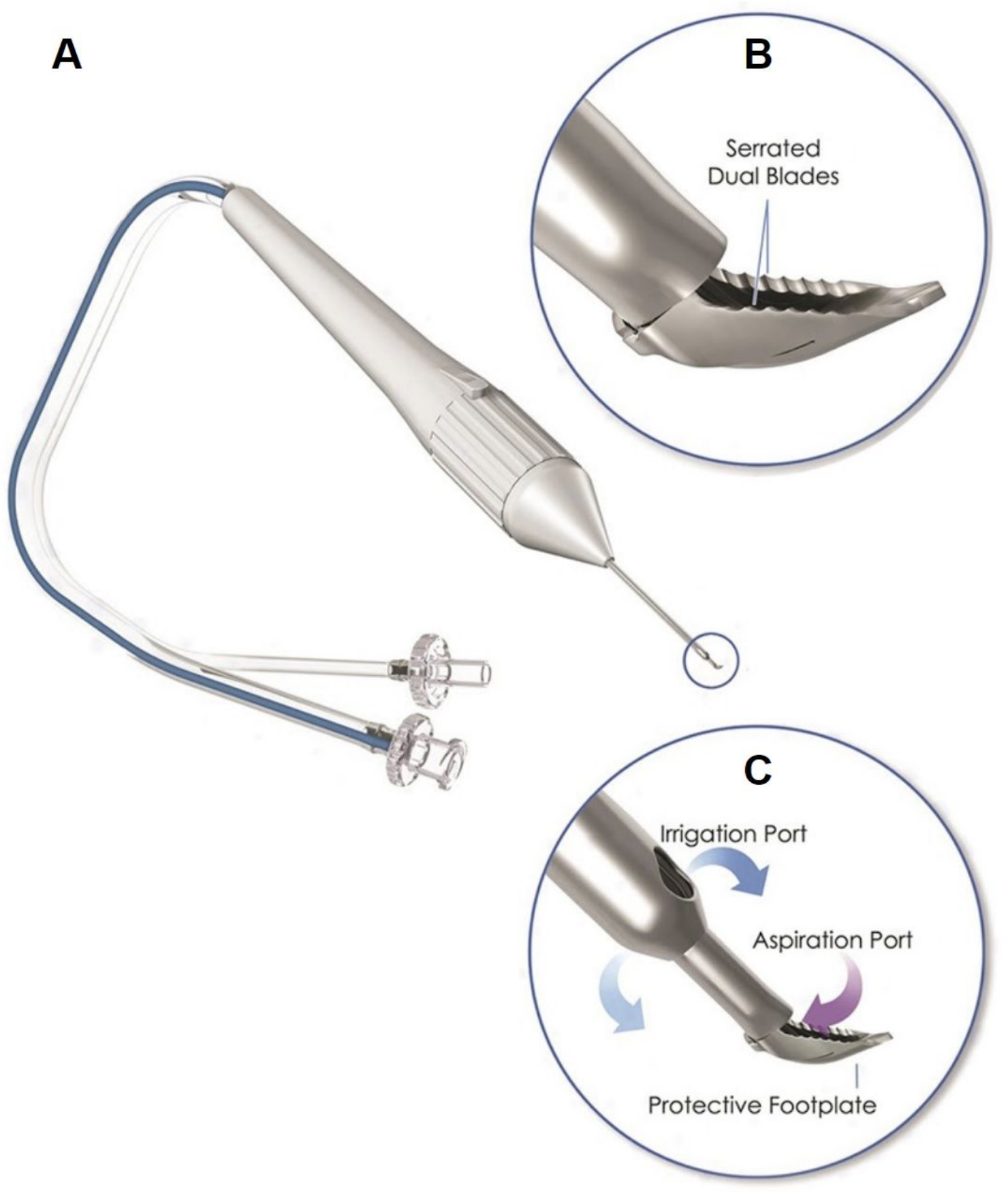

Figure 1: Goniotome surgical system. The Goniotome has two irrigation ports that maintain the chamber (C, blue arrows). The trabecular meshwork is put under stretch by a ramp and excised by a left and a right blade. The trabecular meshwork strip that is generated, blood and debris are aspirated into the tip (C, red arrow) 
Figure 2

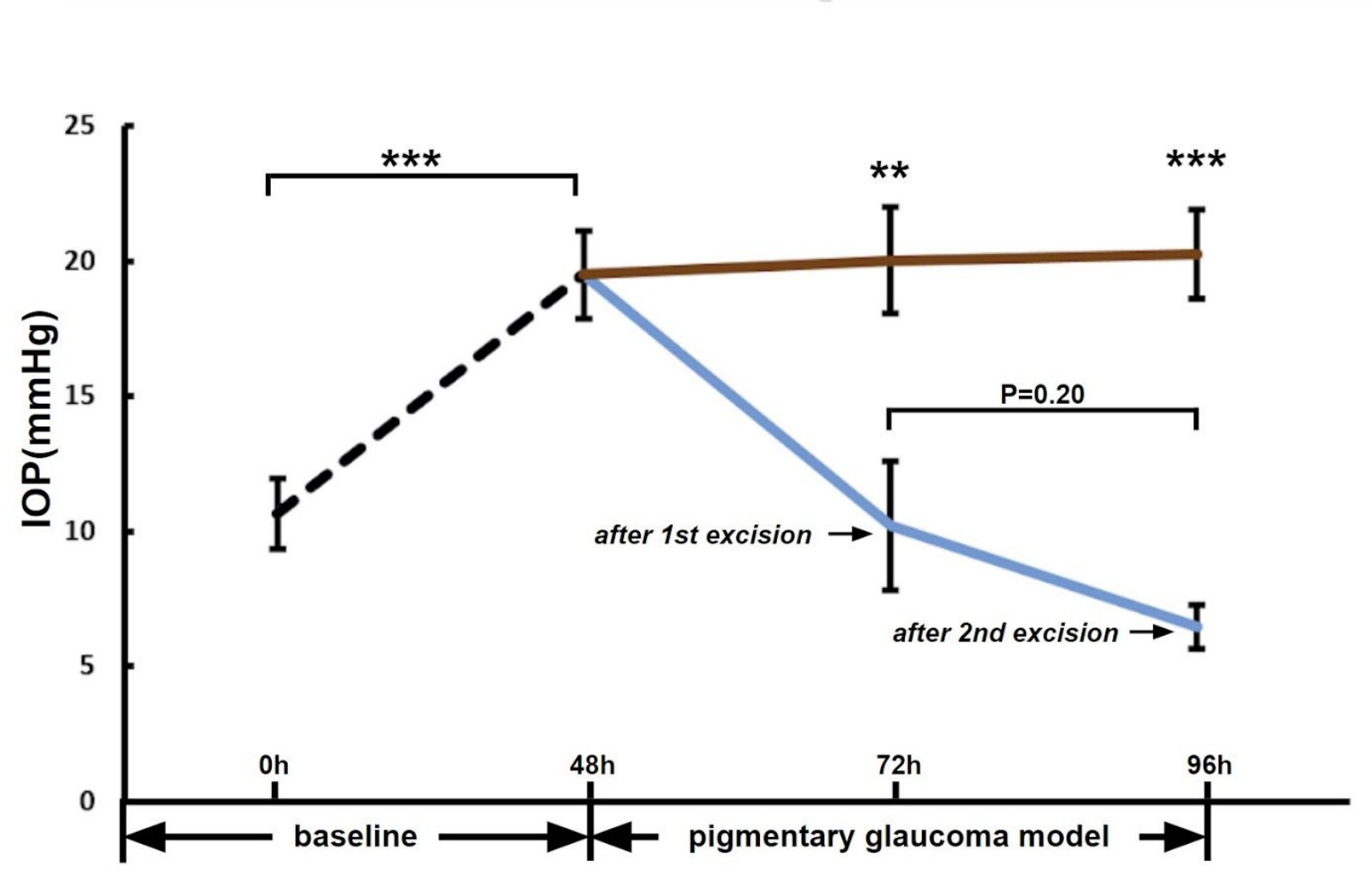

Figure 2: IOP reduction after Goniotome. After 48h pigment perfusion, the IOP was significantly higher compared to baseline IOP $\left(n=15,{ }^{* *} P<0.001\right) .24$ hours after the first $90^{\circ}$ of TM excision with the Goniotome $(G)$, the IOP was significantly lower in $G(n=7)$ than the control $\left(C, n=8,{ }^{* *} P<0.01\right) .24$ hours after the second TM excision, IOP in $G(n=7)$ was significantly lower than $C\left(n=8,{ }^{* *} P<0.001\right)$. 
Figure 3

Goniotome

control

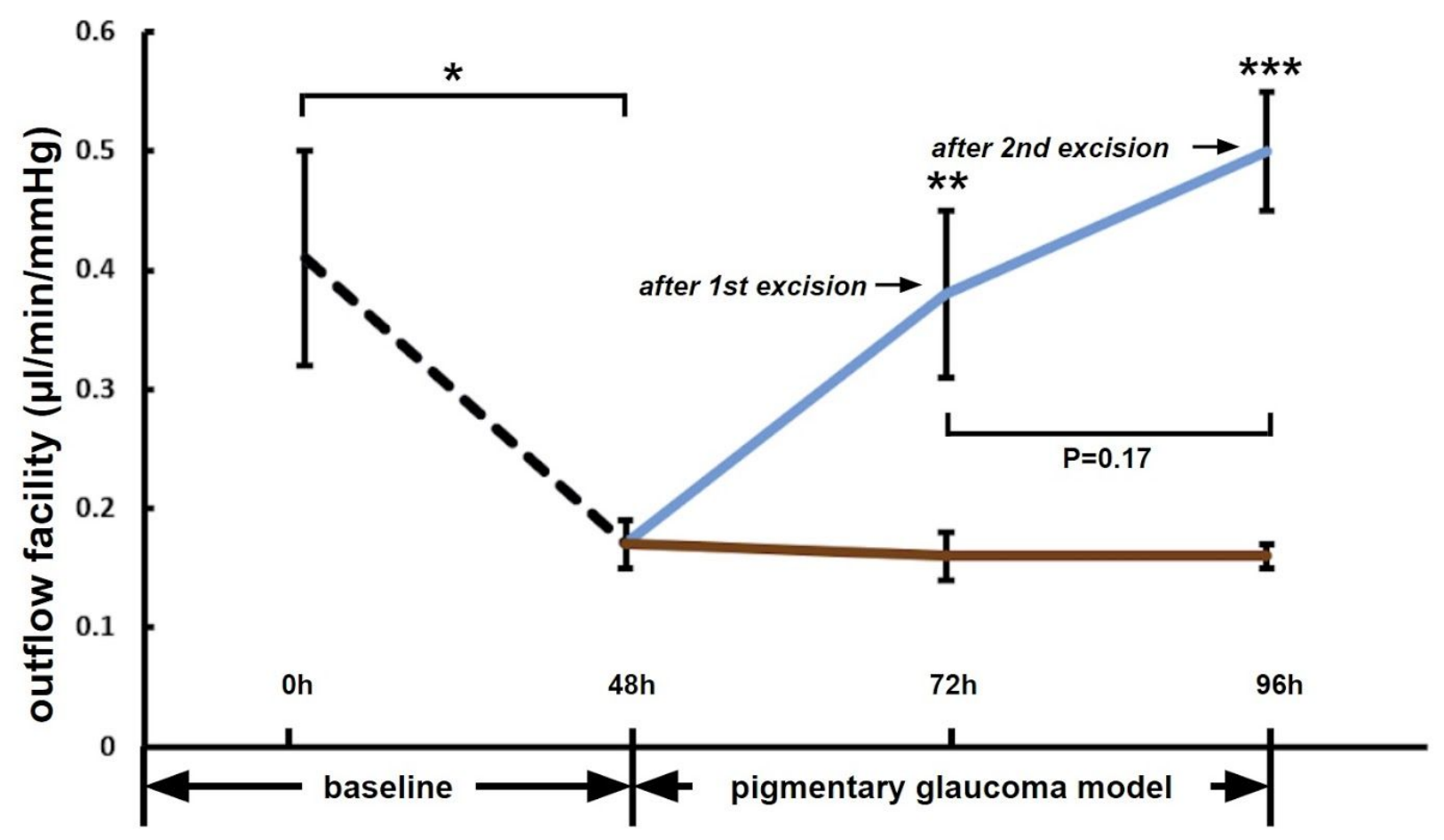

Figure 3: Increased outflow facility after Goniotome. Outflow facility of PG eyes was significantly decreased from baseline $\left(n=15,{ }^{*} P<0.05\right)$ after 48 h pigment perfusion. 24 hours after the first $90^{\circ}$ of TM excision, outflow facility in $G(n=7)$ decreased, compared to $C\left(n=8,{ }^{* *} P<0.01\right) .24$ hours after the second $90^{\circ}$ of excision, outflow facility in $G(n=7)$ was significantly higher than $C\left(n=8,{ }^{* * *} P<0.001\right)$. 


\section{Figure 4}
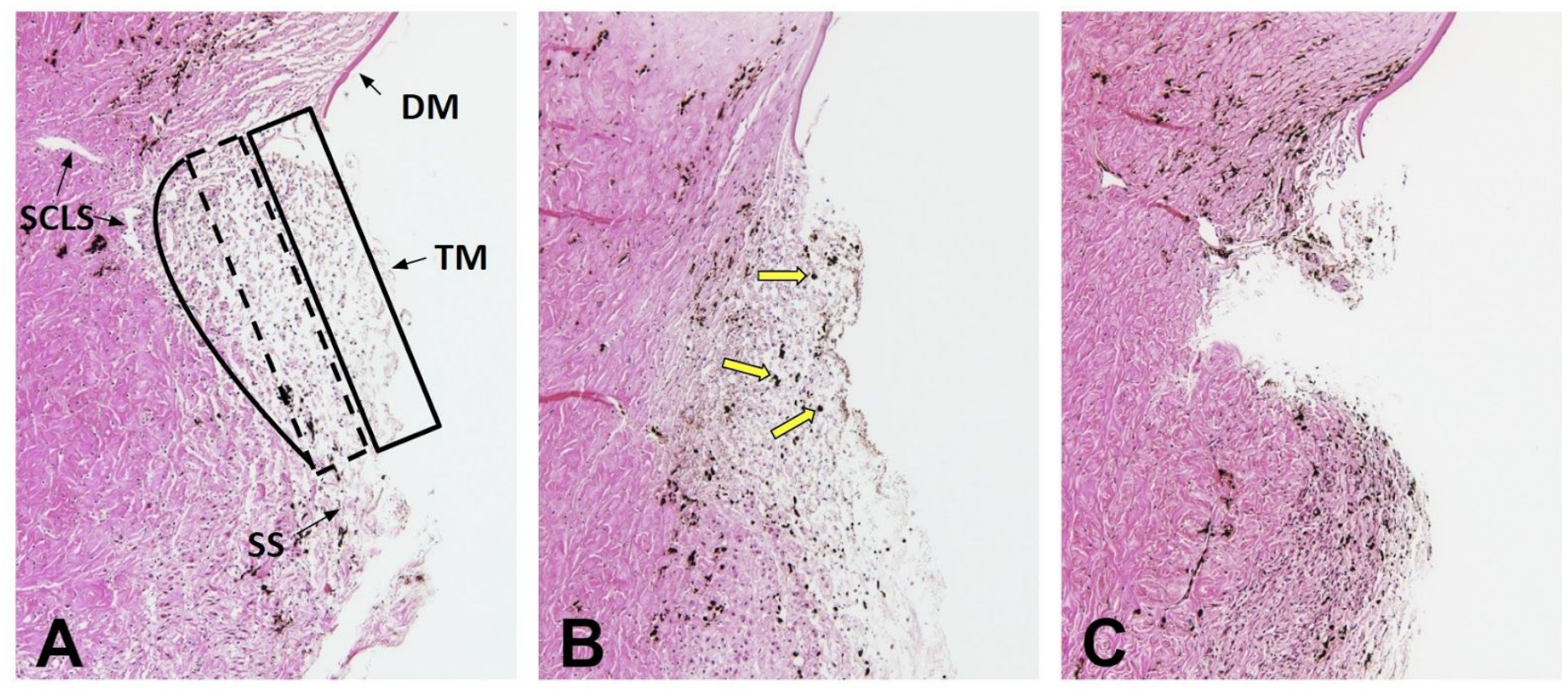

Figure 4: Histology. Normal TM tissue was lightly-pigmented and consisted of three areas: the uveal meshwork (box with solid line, A), the corneoscleral meshwork (box with dashed line, Figure 4A), and the juxtacanalicular meshwork (solid line, A) which adjacent to the inner wall of Schlemm's canal-like segments of the porcine angular aqueous plexus (SCLS). Pigment granules were observed in the TM area (B, yellow arrows), after 48 hours of perfusion with pigment-supplemented media. After Goniotome TM ablation, a full thickness portion of the TM was removed (C). Trabecular meshwork: TM, Schlemm's canal-like segments (SCLS) of the porcine angular aqueous plexus, scleral spur (SS), Descemet's membrane (DM). 